\title{
Análisis psicométrico del Cuestionario de Regulación Conductual de las Emociones en universitarios peruanos
}

\author{
Sergio Dominguez-Lara; Jhonatan S. Navarro-Loli; Candy Ariza-Cruz; \\ Leonardo A. Medrano; Denisse Manrique-Millones
}

Cómo citar este artículo:

Dominguez-Lara, S., Navarro-Loli, J. S., Ariza-Cruz, C., Medrano, L. A., \& Manrique-Millones, D. (2022). Análisis psicométrico del Cuestionario de Regulación Conductual de las Emociones en universitarios peruanos. Acta Colombiana de Psicología, 25(1), 72-86. https://www.doi.org/10.14718/ACP.2022.25.1.6

Recibido, febrero 21/2020; Concepto de evaluación, junio 16/2021; Aceptado, octubre 20/2021

\author{
Sergio Dominguez-Lara ${ }^{1}$ \\ ORCID: http://orcid.org/0000-0002-2083-4278 \\ Universidad Privada San Juan Bautista, Lima, Perú. \\ Universidad de San Martín de Porres, Lima, Perú. \\ Jhonatan S. Navarro-Loli \\ ORCID: http://orcid.org/0000-0001-6264-3157 \\ Universidad de San Martín de Porres, Lima, Perú. \\ Candy Ariza-Cruz \\ ORCID: https://orcid.org/0000-0002-2080-9427 \\ Universidad San Ignacio de Loyola, Lima, Perú. \\ Leonardo A. Medrano \\ ORCID: http://orcid.org/0000-0002-3371-5040 \\ Pontificia Universidad Católica Madre y Maestra, Santiago de los Caballeros, República Dominicana. \\ Universidad Siglo 21, Córdoba, Argentina. \\ Denisse Manrique-Millones \\ ORCID: http://orcid.org/0000-0003-4602-5396 \\ Universidad Científica del Sur, Lima, Perú.
}

\begin{abstract}
Resumen
La presente investigación tuvo como objetivo traducir e identificar las propiedades psicométricas del Behavioral Emotion Regulation Questionnaire (BERQ) en 315 universitarios de Lima, Perú, entre los 16 y 44 años $\left(M_{\text {edad }}=21.31 ; D E_{\text {edad }}=3.39\right.$; 59.7 \% hombres). Para la evaluación se aplicó el BERQ y el Inventario Multicultural de Depresión Estado Rasgo (IMUDER), y las evidencias de validez de la estructura interna se obtuvieron por medio de un análisis factorial confirmatorio y un modelamiento exploratorio de ecuaciones estructurales, mientras que las evidencias de validez en relación con otras variables se obtuvieron a través de un análisis de regresión lineal. Los resultados indican que la estructura pentafactorial se replica en la muestra peruana, que las estrategias adaptativas predicen significativamente el eutrés, y que las estrategias desadaptativas predicen el distrés; además, los valores de confiabilidad fueron aceptables. Al final se discuten aspectos teóricos y prácticos de los hallazgos y la importancia de continuar brindando evidencia para su utilización en diferentes poblaciones y contextos, teniendo en cuenta que esta la primera vez que se analiza una versión en español del BERQ.

Palabras clave: regulación emocional, estrategias conductuales, estudiantes universitarios.
\end{abstract}

\footnotetext{
${ }^{1}$ Vicerrectorado de Investigación de la Universidad Privada San Juan Bautista, Lima, Perú. Av. Juan A. Lavalle S/N, Chorrillos, Perú. sergio.dominguez@upsjb.edu.pe; sdominguezmpcs@gmail.com
} 


\title{
Psychometric analysis of the Behavioral Regulation of Emotions Questionnaire in Peruvian university students
}

\begin{abstract}
The present study aimed to translate and identify the psychometric properties of the Behavioral Emotion Regulation Questionnaire (BERQ) in 315 university students from Lima, Peru, aged 16 to 44 years (Mage = 21.31; SD = 3.39; $59.7 \%$ male). The BERQ and the Multicultural Inventory of Trait State Depression (IMUDER) were administered for the assessment. Evidence of internal structure validity was obtained through confirmatory factor analysis and exploratory structural equation modeling, while evidence of validity in relation to other variables was obtained through linear regression analysis. The results indicate that the pentafactorial structure is replicated in the Peruvian sample; that adaptive strategies significantly predict eutres, and that maladaptive strategies predict distress; in addition, reliability values were acceptable. At the end, theoretical and practical aspects of the findings and the importance of continuing to provide evidence for its use in different populations and contexts are discussed, taking into account that this is the first time that a Spanish version of the BERQ has been analyzed.

Keywords: emotional regulation, behavioral strategies, university students.
\end{abstract}

\section{Introducción}

Estudiar en la universidad conlleva una serie de desafíos que pueden transformarse en una fuente de malestar emocional si no son afrontados adecuadamente, ya que durante la transición a una carrera universitaria aumentan los niveles de estrés crónico y se experimentan los primeros síntomas asociados a trastornos mentales (Cuijpers et al., 2016). De hecho, se estima que un $60 \%$ de los estudiantes que abandonan sus estudios experimentan problemas emocionales, tales como ansiedad, depresión y trastornos adaptativos (Wahed \& Hassan, 2017).

En particular, el afecto es una categoría que implica una valoración, y engloba la respuesta al estrés, el estado de ánimo y la emoción (Gross, 2015; Scherer, 1984). En este orden de ideas, la emoción se caracteriza por ser una experiencia subjetiva acompañada de cambios fisiológicos y comportamentales (Mauss et al., 2005) que pueden ser útiles o perjudiciales para el cumplimiento de un objetivo o meta (Gross, 2015). Esta circunstancia hace necesario que exista una regulación emocional (RE), entendida como los procesos mediante los cuales las personas tienen control e influencia sobre cómo se tienen, experimentan y expresan las emociones (Gross, 2008), siendo motivados por aspectos hedónicos e instrumentales (Tamir, 2016), y dependientes del contexto de la persona (Gross, 2015).
Para el proceso de RE, se tiende a utilizar diversas estrategias que pueden ser variables y flexibles (Aldao et al., 2010), y que podrían ser cognitivas (Garnefski et al., 2001) o conductuales (Kraaij \& Garnefski, 2019). Las estrategias cognitivas han sido ampliamente abordadas en otros estudios (Garnefski et al., 2001; Medrano, Muñoz-Navarro et al., 2016), de manera que es competencia del presente estudio el abordaje de las estrategias conductuales.

Según Kraaij y Garnefski (2019), las estrategias conductuales de RE son: (a) distraerse, una estrategia orientada a la realización de otras actividades con la finalidad de distraerse de la emoción percibida; (b) enfrentar activamente, que se orienta a la ejecución de comportamientos para afrontar el evento; (c) buscar apoyo social, que es el pedir apoyo para hacer frente el evento; (d) retirada, que es alejarse de los eventos y personas; y, por último, (e) ignorar el problema, que es comportarse como si nada hubiera sucedido.

La evidencia previa indica que las estrategias de RE estarían vinculadas a diversas variables, como se ha encontrado respecto a la depresión, la ansiedad o el estrés (Joorman \& Stanton, 2016; Liu \& Thompson, 2017; Völlink, Bolman, Dehue et al., 2013; Völlink, Bolman, Eppingbroek et al., 2013). Sin embargo, si bien los estudios empíricos acerca de las estrategias conductuales de RE son recientes, solo se ha encontrado que las estrategias de retirada e ignorar el problema presentan asociaciones positivas con ansiedad 
y depresión, mientras que las otras estrategias — distraerse, buscar apoyo social, y enfrentar activamente- no evidencian asociación con estos dos aspectos (Kraaij \& Garnefski, 2019).

Por otro lado, las investigaciones realizadas desde el marco del afrontamiento al estrés indican que existen estrategias como la distracción que se asocian al afrontamiento de eventos de la vida valorados como negativos (Joorman \& Stanton, 2016), mientras que el acercamiento activo y la búsqueda de apoyo social son factores protectores contra la depresión, la ideación suicida (Horwitz et al., 2011) y el estrés (Mahmoud et al., 2015), y las estrategias de ignorar y retirada se asocian a procesos vinculados al malestar, como el estrés (Pritchard et al., 2007), la baja autoestima (Strenna et al., 2009), la depresión, la ansiedad, los desórdenes alimenticios y el abuso de sustancias (Aldao et al., 2010; Coiro et al., 2017; Kaminski et al., 2006).

Por tales motivos, es esperable que distraerse, enfrentar activamente y buscar apoyo social se asocien con un modo favorable de manejar eventos estresantes o con variables asociadas al bienestar, mientras que ignorar el problema y retirada se vincularían con una forma desfavorable de lidiar con los problemas, o con variables asociadas al malestar emocional (Joorman \& Stanton, 2016).

Con respecto a la medición de las estrategias conductuales de RE, el Behavioral Emotion Regulation Questionnaire (BERQ; Kraaij \& Garnefski, 2019) es uno de los más importantes en la literatura sobre el tema. Respecto a este instrumento, las primeras evidencias de validez responden a su estructura interna - distraerse, ignorar el problema, retirada, enfrentar activamente, y buscar apoyo social-, las cuales fueron obtenidas mediante de un análisis por componentes principales (ACP), la regla de Kaiser para determinar el número de factores (autovalores $>1$ ), la rotación oblicua (oblimin), y las correlaciones entre las puntuaciones de las dimensiones. Puntualmente, los hallazgos son consistentes con la teoría, dado que se recuperó la estructura pentafactorial, la asociación entre dimensiones es coherente - $\mathrm{p}$. ej. asociación negativa entre enfrentar activamente y buscar apoyo social con ignorar el problema-, y porque se encuentran relaciones con las estrategias cognitivas de regulación emocional (Garnefski et al., 2001). No obstante, es posible mejorar algunos procedimientos observados en la metodología.

Por ejemplo, el uso del ACP como análogo a un análisis factorial exploratorio (AFE) no es adecuado según la literatura especializada, debido a que ambos análisis persiguen objetivos diferentes (Lloret-Segura et al., 2014). Adicionalmente, el ACP sobreestima la magnitud de los parámetros factoriales, dado que no separa la varianza común de la varianza específica, y la regla de Kaiser tiende a sobreestimar el número de factores (Lloret-Segura et al., 2014; Zwick \& Velicer, 1986). Por tales motivos, estos procesos pueden ocasionar problemas en las inferencias que se realicen usando las puntuaciones del instrumento, por lo que sería recomendable un marco analítico más potente.

En este sentido, una alternativa viable y coherente con el desarrollo actual de los estudios psicométricos podría ser el modelamiento exploratorio de ecuaciones estructurales (ESEM; Asparouhov \& Muthén, 2009), pues este considera la existencia de cargas secundarias $— o$ cargas factoriales asociadas a un factor distinto del teórico- que suelen ser significativas en modelos factorialmente complejos, y cuya existencia podría ayudar a comprender en mayor medida la estructura interna del instrumento. Del mismo modo, sería útil trabajar con un modelo de contraste, con el fin de comparar los resultados encontrados en el modelo original con los de un modelo teóricamente incoherente que asuma el rol de una hipótesis nula — para este caso, que todos los ítems sean influidos por un solo factor-. Por último, el uso de las puntuaciones directas para establecer asociaciones entre dimensiones podría contener error de medida, por lo que sería conveniente trabajar a nivel de constructo con correlaciones interfactoriales - que suelen ser de mayor magnitud que las correlaciones entre puntuaciones-.

Con base en lo expuesto, y teniendo en cuenta que en el contexto peruano no existen instrumentos de evaluación de las estrategias conductuales de RE, el presente estudio tuvo como objetivo traducir e identificar las propiedades psicométricas del BERQ, ya que estas estrategias se asocian a aspectos positivos y negativos del comportamiento humano en el área social y en la salud física y mental, de manera que pueden tener consecuencias directas sobre aspectos académicos del estudiante universitario.

En ese sentido, se analizaron las evidencias de validez del instrumento respecto a su estructura interna — con el ESEM como herramienta metodológica-, con lo cual se esperaba que la estructura pentafactorial recibiera el respaldo adecuado (hipótesis 1), que las cargas secundarias no fueran de magnitud significativa (hipótesis 2), y que se encontrara un mejor modelo que el alternativo (hipótesis 3); 
por otra parte, las evidencias de validez con respecto a otras variables —específicamente, la validez predictiva - se exploraron considerando la influencia de las estrategias del BERQ sobre una medida de eutrés - afectividad positiva- $\mathrm{y}$ distrés - afectividad negativa-, con lo cual se esperaba que distraerse, enfrentar activamente y buscar apoyo social fueran predictores positivos del eutrés y negativos del distrés (hipótesis 4), y que ignorar el problema y retirada tuvieran un patrón inverso (hipótesis 5). En cuanto a la confiabilidad, se esperaba que la magnitud de confiabilidad del constructo y puntuaciones fuera aceptable en todos los casos (> .70; hipótesis 6).

\section{Método}

\section{Diseño}

La presente investigación se realizó a partir de un diseño instrumental (Ato et al., 2013), debido a que se exploró respecto a las propiedades psicométricas de un instrumento de medición.

\section{Participantes}

La población fue la conformada por estudiantes de una universidad privada de Lima Metropolitana, Perú, y la muestra se seleccionó con un muestreo no probabilístico. En total, la muestra estuvo compuesta por 315 participantes de entre 16 y 44 años de edad $(M=21.31 ; D E=3.39)$; específicamente, el $59.7 \%(n=188)$ era de sexo femenino, el $56.8 \%(n=179)$ nació en el departamento de Lima, y, en mayor proporción, los participantes pertenecían al quinto ciclo (15.6\%), octavo ciclo (14.9\%), décimo ciclo (14\%), séptimo ciclo (11.1\%) y tercer ciclo (10.8\%) de las áreas profesionales de ingenierías (31.43\%), administración y ciencias empresariales (24.44\%), humanidades (12.38\%), psicología (9.52\%), arquitectura $(6.35 \%)$ y otras $(16.19 \%)$.

\section{Instrumentos}

Behavioral Emotion Regulation Questionnaire (BERQ, Kraaij \& Garnefski, 2019)

El BERQ es un instrumento conformado por 20 ítems con formato de respuesta tipo Likert —de 1 = "Casi nunca o nunca" a 5 = "Casi siempre o siempre"-, distribuidos en cinco dimensiones que representan cada estrategia de regulación conductual de las emociones - distraerse $\left(\alpha_{\text {original }}=.86\right)$, retirada $\left(\alpha_{\text {original }}=.93\right)$, enfrentar activamente $\left(\alpha_{\text {original }}=.91\right)$, buscar apoyo social $\left(\alpha_{\text {original }}=.91\right)$ e ignorar el problema $\left(\alpha_{\text {original }}=.89\right)$ - , que pueden ser utilizadas ante diferentes tipos de eventos estresantes (afrontamiento disposicional) $o$ ante un evento en específico (afrontamiento situacional).

Inventario Multicultural de Depresión Estado-Rasgo (Moscoso et al., 2012)

Este inventario es un instrumento de screening adaptado a población latinoamericana —incluyendo a Perú-, conformado por 24 ítems que tienen como fin identificar sintomatología asociada a la depresión. Específicamente, cuenta con una sección de "estado" (12 ítems), que mide la presencia o ausencia de sintomatología asociada a la depresión en el momento actual, y una sección de "rasgo" (12 ítems), que mide la frecuencia de ocurrencia de la sintomatología depresiva. Además, en ambas secciones se encuentran los factores de eutrés (afecto positivo) y distrés (afecto negativo). En el presente estudio se utilizó la sección "rasgo", sobre la cual se llevó a cabo un análisis factorial confirmatorio, donde se obtuvieron resultados aceptables en cuanto a los índices de ajuste (CFI $=.961$; RMSEA $=.072$ [IC $90 \% .056-.088]$; WRMR $=.893) \mathrm{y}$ confiabilidad $\left(\alpha_{\text {eutrés }}=.758 ; \alpha_{\text {distrés }}=.695\right)$, aunque prescindiendo del ítem 8 - "Pienso que los fracasos son parte de la vida"-, perteneciente al eutrés.

\section{Procedimiento}

La traducción fue un proceso sistematizado que siguió las recomendaciones de la literatura especializada (Hambleton, 2005; Muñiz et al., 2013). La fase inicial consistió en obtener la autorización vía correo electrónico de las creadoras del BERQ para el proceso de traducción al español. En la segunda fase, se entregó el BERQ - instrucción, ítems y alternativas de respuesta - a seis profesionales - tres psicólogos y tres traductores - de nacionalidad peruana que tuvieran como lengua nativa el español y que contaran con dominio avanzado del idioma inglés, con la finalidad de obtener versiones que tuvieran un balance entre términos psicológicos y de uso cotidiano. En la tercera fase, las seis versiones obtenidas fueron sistematizadas por dos de los autores, $\mathrm{y}$, cuando había discrepancias entre versiones, se solicitó la colaboración de una investigadora con dominio del idioma inglés para identificar aquellos aspectos culturales 
inherentes a la versión original, con el fin de que sugiriera cómo podrían ajustarse al idioma español. Como resultado de este proceso, se obtuvo una versión del BERQ que, en la cuarta fase, se presentó a 10 estudiantes universitarios que evaluaron la claridad y comprensión de los ítems. Tras este procedimiento, al no presentar observaciones respecto al contenido de los ítems, se obtuvo la versión final del BERQ en español.

Por otra parte, la recogida de información para la validación de la versión en español de la prueba se realizó a través de un formulario virtual creado en Google Forms, enviado a través de correo electrónico, en el que inicialmente se brindaba información sobre los investigadores, el objetivo del estudio y la evaluación, el tiempo promedio de evaluación, y las condiciones tecnológicas necesarias para proceder al llenado del cuestionario. Al acceder al formulario, el estudiante podía aceptar o no a participar mediante la aceptación del consentimiento informado, en el que se indicaba el anonimato del proceso, que los datos solo se utilizarían con fines de la investigación y que el estudiante no obtendría beneficio monetario o académico por su participación. Cabe recalcar que toda la evaluación se realizó siguiendo las pautas éticas de The Standards for Educational and Psychological Testing (American Educational Research Association, American PsychologicalAssociation \& National Council on Measurement in Education, 2014).

\section{Análisis de datos}

El análisis descriptivo de los datos se realizó considerando la asimetría y curtosis, en las cuales valores entre $-2 \mathrm{y}+2$ indicaban una distribución que se aproxima a la normal (Gravetter \& Wallnau, 2014; Pérez \& Medrano, 2010). Asimismo, se empleó el coeficiente de Mardia (1970) para el análisis de normalidad multivariada $(<70$; Rodríguez \& Ruiz, 2008).

Por otra parte, el análisis de la estructura interna se realizó a partir de un análisis factorial confirmatorio (AFC) $\mathrm{y}$ un modelamiento exploratorio de ecuaciones estructurales (ESEM), mediante el método de extracción mínimos cuadrados ponderados con varianza ajustada (WLSMv; por sus siglas en inglés), con base en la matriz de correlaciones policóricas, y con rotación geomin $(\varepsilon=.05$; Asparouhov \& Muthen, 2009) para el caso del ESEM. Adicionalmente, se analizó un modelo de cinco factores (original) y un modelo bifactor, a modo de contraste, el cual considera que todos los ítems son influidos por un factor general. Para ello, se invirtieron las puntuaciones de ignorar el problema y retirada para que todos los ítems fueran en la misma dirección.

La viabilidad de los modelos se determinó con diferentes fuentes de información. La primera se enfocó en la magnitud de diversos índices de ajuste, como el CFI ( $>$.90; McDonald \& Ho, 2002), el límite superior del intervalo de confianza (IC) del RMSEA ( $<.10$; West et al., 2012), y el WRMR (<1.00; DiStefano et al., 2018). La segunda fuente fue la magnitud de las cargas factoriales, donde se esperaban valores $>.60$ (Dominguez-Lara, 2018), y la varianza media extraída por factor (vME > .50; Hair et al., 2010). La tercera fuente se enfocó en la diferenciación empírica de las dimensiones, mediante la comparación entre la VME por factor y el cuadrado de la correlación interfactorial $\left(\phi^{2}\right.$; varianza compartida entre factores), donde se esperaba que el primer indicador fuera mayor (Fornell \& Larcker, 1981). Luego de ello, en vista de que el ESEM contempla la existencia de cargas secundarias, se implementó el índice de simplicidad factorial (ISF; Fleming \& Merino, 2005), donde se esperaban valores $>.70$ para concluir que el ítem es influido en mayor medida por un solo factor. Todos los procedimientos se llevaron a cabo con el software Mplus, versión 7 (Muthén \& Muthén, 1998-2015).

Por último, para el caso del modelo bifactor se consideró el coeficiente omega jerárquico general $\left(\omega_{h}\right.$; Zinbarg et al., 2006) — que indica la proporción de varianza total que explica el factor general一, el omega jerárquico por dimensión $\left(\omega_{h s}\right)$ - o la proporción de varianza verdadera explicada por los factores específicos en presencia del factor general—, y la varianza común explicada (ECV; Sijtsma, 2009) por el factor general. El factor general explica en mayor grado la varianza de los ítems si el ECV y el $\omega_{h}$ son mayores que 70 (Rodríguez et al., 2016) y el $\omega_{h s}<.30$ (Smits et al., 2015).

De otro lado, en el AFC se exploró la presencia de potenciales malas especificaciones (Saris et al., 2009), lo que hace referencia a una situación de sobre-parametrización — cuando se especifica un parámetro cuyo valor poblacional es cero- 0 infra-parametrización — cuando no se especifica un parámetro cuyo valor poblacional es diferente de cero- (Hu \& Bentler, 1998). En este caso, se pensó que podrían estar asociadas a cargas cruzadas - es decir, cargas secundarias no especificadas - La información se recabó considerando índices de modificación (IM) significativos $\left(\chi^{2}>10\right)$, con un módulo especializado (Dominguez-Lara \& Merino-Soto, 2018). 
Adicional a esto, la identificación de las evidencias de validez predictiva se realizó mediante un análisis de regresión lineal, donde las estrategias conductuales de regulación emocional fueron consideradas predictoras de medidas de eutrés y distrés. Acá, la significancia se abordó desde un enfoque de magnitud del efecto, en el que los coeficientes $\beta$ mayores que 20 (Ferguson, 2009) fueron significativos, mientras que el $\mathrm{R}^{2} \longrightarrow \mathrm{O}$ la varianza del distrés o eutrés explicadas por las estrategias - se interpretó considerando valores menores que .02 como insignificantes, entre .02 y .13 como pequeños, entre .13 y .26 como medianos, y mayores que .26 como grandes (Ellis, 2010).

En cuanto a la estimación de la confiabilidad, se tomó en cuenta el coeficiente $\omega$ para valorar el constructo $(>.70$; Hunsley \& Marsh, 2008), y el coeficiente $\alpha$ (> .70; Ponterotto $\&$ Charter, 2009) para las puntuaciones, previo análisis de la tau-equivalencia o equivalencia estadística de las cargas factoriales al interior de un factor. Y, por último, y con fines descriptivos, se comparó los coeficientes $\alpha$ de hombres y mujeres con un método basado en intervalos de confianza (IC; Dominguez-Lara et al., 2018).

\section{Resultados}

\section{Estadísticos descriptivos}

Se identificó que la asimetría y curtosis de los ítems del BERQ tienen una distribución que se aproxima a la normalidad univariada, mientras que, para el caso de la normalidad multivariada, se obtuvo un coeficiente de 93.501, lo cual excede los criterios establecidos inicialmente.

\section{Evidencias de validez de la estructura interna}

Los índices de ajuste del AFC (véase Tabla 2) para la estructura de cinco factores estuvieron cerca del límite permitido (CFI $=.910$, RMSEA $=.091($ IC $90 \% .083-.099) \mathrm{y}$ WRMR $=1.387)$, la mayoría de las cargas factoriales tuvieron magnitudes aceptables $(\geq .60)$, la VME estuvo por encima de lo esperado, y la diferenciación entre las dimensiones recibió respaldo $\left(\mathrm{VME}>\phi^{2}\right)$.

Con respecto a las malas especificaciones, se detectaron 30 potenciales casos $\left(\chi^{2}>10\right)$, de los cuales 15 se confirmaron como cargas cruzadas significativas no especificadas

Tabla 1.

Medidas de tendencia central y dispersión de los items del BERQ

\begin{tabular}{lcccc}
\hline \multicolumn{1}{c}{ Ítem } & $M$ & $D E$ & $\mathrm{~g} 1$ & $\mathrm{~g} 2$ \\
\hline 1. Me dedico a otras actividades que no estén relacionadas a lo que sucede. & 1.524 & 1.050 & 0.766 & 0.078 \\
2. Evito a las personas. & 1.063 & 1.032 & 1.130 & 0.917 \\
3. Trato de hacer algo al respecto. & 1.952 & 1.109 & 0.306 & -0.638 \\
4. Busco que alguien me consuele. & 1.057 & 1.007 & 0.862 & 0.252 \\
5. Sigo adelante y pretendo que no pasó nada. & 1.781 & 1.204 & 0.385 & -0.856 \\
6. Dejo de lado mis preocupaciones haciendo otra cosa. & 1.784 & 1.096 & 0.408 & -0.658 \\
7. Me retiro. & 0.911 & 0.950 & 1.120 & 1.061 \\
8. Me pongo a trabajar sobre ello. & 1.937 & 1.106 & 0.225 & -0.683 \\
9. Pido consejo a alguien. & 1.806 & 1.107 & 0.191 & -0.827 \\
10. Lo reprimo y pretendo que nunca sucedió. & 1.190 & 1.195 & 1.024 & 0.169 \\
11. Hago otras cosas para distraerme. & 2.025 & 1.108 & 0.218 & -0.855 \\
12. Me aíslo. & 0.981 & 1.064 & 1.170 & 0.895 \\
13. Tomo medidas para enfrentarlo. & 2.000 & 1.120 & 0.192 & -0.782 \\
14. Comparto mis sentimientos con alguien. & 1.654 & 1.130 & 0.405 & -0.686 \\
15. Me comporto como si nada estuviera pasando. & 1.429 & 1.160 & 0.697 & -0.353 \\
16. Me dedico a una actividad que me haga sentir bien. & 2.340 & 1.140 & -0.190 & -0.911 \\
17. Me alejo de los demás. & 1.006 & 1.067 & 1.188 & 0.893 \\
18. Hago lo que sea necesario para afrontarlo. & 1.984 & 1.132 & 0.164 & -0.807 \\
19. Busco alguien que me pueda apoyar. & 1.670 & 1.093 & 0.361 & -0.590 \\
20. Bloqueo lo que está sucediendo. & 1.044 & 0.960 & 0.889 & 0.422 \\
\hline
\end{tabular}

Nota. $M=$ media; $D E$ = desviación estándar; g1 = asimetría; $\mathrm{g} 2$ = curtosis. 
(infra-parametrización), lo cual sugiere modelar los ítems 3 y 13 en distraerse; los ítems 1, 4, 5, 16 y 20 en retirada; los ítems 4, 6, y 16 en enfrentar activamente; el ítem 16 en buscar apoyo social; y los ítems 6, 7, 16 y 17 en ignorar el problema.

Tabla 2.

Análisis factorial confirmatorio del BERQ en estudiantes universitarios

\begin{tabular}{|c|c|c|c|c|c|}
\hline & $\mathrm{F} 1$ & $\mathrm{~F} 2$ & F3 & $\mathrm{F} 4$ & F5 \\
\hline Ítem 3 & .716 & & & & \\
\hline Ítem 8 & .772 & & & & \\
\hline Ítem 13 & .872 & & & & \\
\hline Ítem 18 & .887 & & & & \\
\hline Ítem 4 & & .550 & & & \\
\hline Ítem 9 & & .899 & & & \\
\hline Ítem 14 & & .746 & & & \\
\hline Ítem 19 & & .850 & & & \\
\hline Ítem 1 & & & .460 & & \\
\hline Ítem 6 & & & .657 & & \\
\hline Ítem 11 & & & .812 & & \\
\hline Ítem 16 & & & .734 & & \\
\hline Ítem 5 & & & & .611 & \\
\hline Ítem 10 & & & & .762 & \\
\hline Ítem 15 & & & & .677 & \\
\hline Ítem 20 & & & & .752 & \\
\hline Ítem 2 & & & & & .647 \\
\hline Ítem 7 & & & & & .500 \\
\hline Ítem 12 & & & & & .876 \\
\hline Ítem 17 & & & & & .876 \\
\hline VME & .664 & .598 & .460 & .495 & .551 \\
\hline F1 & 1 & .137 & .171 & .008 & .072 \\
\hline $\mathrm{F} 2$ & .370 & 1 & .115 & .024 & .024 \\
\hline F3 & .414 & .339 & 1 & .181 & .011 \\
\hline $\mathrm{F} 4$ & -.087 & -.154 & .425 & 1 & .328 \\
\hline F5 & -.268 & -.156 & .106 & .573 & 1 \\
\hline
\end{tabular}

Nota. F1 = enfrentar activamente; F2 = buscar apoyo social; F3 = distraerse; F4 = ignorar el problema; F5 = retirada; $\mathrm{VME}=$ varianza media extraída; debajo de la diagonal = correlaciones interfactoriales; encima de la diagonal = varianza compartida entre factores.

Respecto al análisis ESEM (véase Tabla 3), la estructura de cinco factores obtuvo resultados satisfactorios ( $\mathrm{CFI}=.976$, RMSEA $=.059$ [IC $90 \% .048-.071]$ y WRMR $=.511$ ), las cargas factoriales fueron $\geq .60$, a excepción de pocos ítems (p. ej., ítem 7), y la vME por factor fue aceptable en todos los casos.

Tabla 3.

Análisis ESEM del BERQ en estudiantes universitarios

\begin{tabular}{|c|c|c|c|c|c|c|}
\hline & F1 & F2 & F3 & F4 & F5 & ISF \\
\hline Ítem 3 & .703 & .005 & .104 & -.230 & .040 & .858 \\
\hline Ítem 8 & .724 & .066 & .076 & .008 & -.152 & .926 \\
\hline Ítem 13 & .755 & .040 & .290 & -.038 & -.022 & .842 \\
\hline Ítem 18 & .772 & .121 & .175 & .036 & -.130 & .880 \\
\hline Ítem 4 & -.095 & .698 & .054 & -.022 & .219 & .866 \\
\hline Ítem 9 & .192 & .793 & .115 & -.058 & -.028 & .866 \\
\hline Ítem 14 & -.087 & .676 & .210 & -.167 & -.126 & .786 \\
\hline Ítem 19 & .092 & .789 & .093 & -.014 & -.14 & .930 \\
\hline Ítem 1 & -.054 & .079 & .447 & .052 & .236 & .709 \\
\hline Ítem 6 & -.114 & .020 & .666 & .229 & -.076 & .831 \\
\hline Ítem 11 & .104 & -.031 & .732 & .133 & .078 & .923 \\
\hline Ítem 16 & .043 & .046 & .752 & -.062 & -.135 & .945 \\
\hline Ítem 5 & .005 & -.162 & .240 & .584 & -.061 & .754 \\
\hline Ítem 10 & -.009 & .032 & .010 & .725 & .134 & .957 \\
\hline Ítem 15 & .053 & -.042 & .052 & .595 & -.005 & .981 \\
\hline Ítem 20 & .016 & .102 & .029 & .645 & .227 & .841 \\
\hline Ítem 2 & -.071 & -.061 & .214 & .063 & .624 & .841 \\
\hline Ítem 7 & -.129 & .089 & .094 & .272 & .307 & .452 \\
\hline Ítem 12 & .087 & -.029 & -.095 & .165 & .854 & .928 \\
\hline Ítem 17 & -.059 & .002 & -.060 & .131 & .807 & .955 \\
\hline VME & .546 & .549 & .436 & .409 & .466 & \\
\hline F1 & 1 & & & & & \\
\hline $\mathrm{F} 2$ & .115 & 1 & & & & \\
\hline F3 & .221 & .193 & 1 & & & \\
\hline $\mathrm{F} 4$ & -.076 & -.097 & .185 & 1 & & \\
\hline F5 & -.134 & -.038 & -.015 & .293 & 1 & \\
\hline$\alpha$ & .860 & .824 & .728 & .742 & .722 & \\
\hline IC $95 \%$ & $\begin{array}{l}.828 \\
.886\end{array}$ & $\begin{array}{l}.785 \\
.856\end{array}$ & $\begin{array}{l}.672 \\
.776\end{array}$ & $\begin{array}{l}.688, \\
.788\end{array}$ & $\begin{array}{l}.665 \\
.771\end{array}$ & \\
\hline$\omega$ & .828 & .829 & .749 & .733 & .759 & \\
\hline
\end{tabular}

Nota. $\mathrm{F} 1$ = enfrentar activamente; F2 = buscar apoyo social; F3 = distraerse; F4 = ignorar el problema; F5 = retirada. En negrilla $=$ cargas principales; $\mathrm{VME}=$ varianza media extraída; $\alpha=$ coeficiente alfa; $\omega=$ coeficiente omega.

En cuanto al modelo de contraste (bifactor), si bien los índices de ajuste fueron adecuados $(\mathrm{CFI}=.976$; $\mathrm{RMSEA}=.059$ [IC $90 \% .048-.071$ ]; WRMR $=.511)$, la magnitud del $\omega_{h}(.371)$ 
Tabla 4.

Capacidad predictiva de las estrategias del BERQ sobre el distrés y el eutrés

\begin{tabular}{|c|c|c|c|c|c|c|}
\hline & \multicolumn{3}{|c|}{ Distrés } & \multicolumn{3}{|c|}{ Eutrés } \\
\hline & $\mathrm{B}$ & $t$ & $\beta$ & $\mathrm{B}$ & $t$ & $\beta$ \\
\hline Distraerse & -.194 & -3.520 & -.215 & .155 & 2.971 & .182 \\
\hline Enfrentar activamente & -.116 & -2.802 & -.147 & .168 & 4.305 & .227 \\
\hline Buscar apoyo social & .088 & 2.055 & .105 & .048 & 1.197 & .061 \\
\hline Ignorar el problema & .186 & 3.437 & .197 & -.057 & -1.013 & -.064 \\
\hline Retirada & .400 & 7.615 & .414 & -.335 & -6.745 & -.368 \\
\hline
\end{tabular}

Nota. En negrilla los coeficientes beta significativos.

y del ECV (.263) indican que la influencia de un factor general sobre todos los ítems no recibe respaldo empírico.

Entonces, considerando estos resultados, se puede afirmar que la estructura pentafactorial recibe respaldo empírico (hipótesis 1); que las cargas secundarias no fueron de magnitud significativa, de manera que no representan una potencial complejidad factorial (hipótesis 2), excepto el ítem 7 - "Me retiro"-; y que el modelo es significativamente superior a un modelo alternativo y carente de sentido teórico (hipótesis 3).

\section{Evidencias de validez predictiva}

El análisis de regresión lineal indica que las estrategias conductuales de regulación emocional predicen significativamente el distrés $\left(\mathrm{R}^{2}=.294\right)$ y el eutrés $\left(\mathrm{R}^{2}=.288\right)$, en ambos casos a un nivel considerado grande. De forma específica, solo las estrategias distraerse, ignorar el problema y retirada predicen significativamente el distrés, y distraerse, enfrentar activamente y retirada son predictoras significativas del eutrés (véase Tabla 4), lo que da cumplimiento parcial a las hipótesis 4 y 5 .

\section{Confiabilidad}

Se realizó el análisis de la tau-equivalencia tomando como modelo de base el provisto por el CFA. A partir de esto, y una vez realizada la restricción de igualdad de cargas factoriales al interior de cada factor $-\mathrm{p}$. ej., las cargas factoriales de retirada se modelaron como si fueran estadísticamente similares-, los índices de ajuste no fueron aceptables (CFI $=.862$, RMSEA $=.108[$ IC $90 \%$ .100-.115] y WRMR $=1.758$ ), lo cual impidió la estimación del coeficiente $\alpha$ libre de sesgo. Por su lado, el coeficiente $\omega$ obtenido en cada caso presentó magnitudes que permiten concluir que la varianza verdadera asociada al constructo es significativa (véase Tabla 3), lo cual brinda soporte a la hipótesis 6. Por último, no se encontró diferencias significativas entre hombres y mujeres respecto a la magnitud del coeficiente $\alpha$ (véase Tabla 5).

Tabla 5.

Análisis comparativo de la confiabilidad de las estrategias del BERQ entre hombres y mujeres

\begin{tabular}{lccc}
\hline & $\alpha_{\text {hombres }}$ & $\alpha_{\text {mujeres }}$ & $\mathrm{IC}_{\text {diferencia }}$ \\
\hline Enfrentar activamente & .845 & .864 & $-.078, .034$ \\
Buscar apoyo social & .826 & .817 & $-.060, .074$ \\
Distraerse & .711 & .729 & $-.129, .084$ \\
Ignorar el problema & .719 & .753 & $-.141, .062$ \\
Retirada & .707 & .725 & $-.131, .085$ \\
\hline
\end{tabular}

\section{Discusión}

La RE se asocia a una amplia variedad de trastornos psicopatológicos. Su naturaleza transdiagnóstica ha llevado a reconocer esta variable como tema de central importancia para diferentes modelos teóricos (Barlow et al., 2004). Inicialmente, el foco de investigación se centró en el papel de los procesos cognitivos involucrados en la RE, ya que la atención e interpretación cognitiva que realicemos de los eventos determinará el tipo de emoción experimentada y modulará la respuesta emocional (Joormann et al., 2010); no obstante, solo recientemente se ha comenzado a analizar las estrategias conductuales utilizadas para regular una respuesta emocional, y es en este marco que se desarrolló el BERQ (Kraaij \& Garnefski, 2019).

Tal como argumenta Garnefski et al. (2001), las estrategias cognitivas y conductuales de la regulación emocional deberían considerarse de forma separada, ya que pensar y actuar son procesos diferentes que se utilizan en momentos 
distintos. Teniendo esto en cuenta, para desarrollar el BERQ los autores se basaron en instrumentos previos que examinaban estrategias de afrontamiento al estrés, y sumaron nuevas dimensiones. A partir de ello, se propuso un modelo de cinco factores subyacentes: (a) distracción, que refiere a la estrategia de redirigir la atención hacia estímulos o actividades más agradables; (b) retirada, que refiere a la conducta de evadir situaciones y contactos sociales que generen emociones displacenteras; (c) enfrentamiento activo, que involucra conductas dirigidas a lidiar con el evento estresante; (d) búsqueda de apoyo social, como, por ejemplo, pedir apoyo y consejos para hacer frente al evento estresante; y, por último, (e) ignorar el problema, es decir, comportarse como si nada hubiera pasado (Kraaij \& Garnefski, 2019).

El presente trabajo constituye el primer estudio psicométrico de una versión del BERQ en idioma español, y, en términos generales, los resultados obtenidos son coherentes con las hipótesis planteadas y los estudios antecedentes.

Respecto a las evidencias de validez de estructura interna, con los resultados obtenidos fue posible corroborar el modelo de cinco factores formulado inicialmente. A diferencia del estudio original, no se utilizó ACP, ya que este método sobreestima la magnitud de los parámetros factoriales; no obstante, utilizar un procedimiento más potente como el ESEM no modificó la cantidad de factores subyacentes identificados. De hecho, los resultados del análisis ESEM indicaron que el modelo de cinco factores presenta un ajuste satisfactorio y superior a los modelos rivales. Asimismo, los ítems que componen cada una de las cinco escalas presentan en su mayoría saturaciones factoriales superiores a 60 .

Una excepción para considerar es el ítem 7-"Me retiro"-, que presentó una saturación inferior a la recomendada por la literatura (valores por debajo de .40). Probablemente, la expresión en español no refleja con claridad el componente de evasión que intenta reflejar el reactivo original. Quizás expresiones alternativas como "me escapo" o "huyo de la situación" puedan mostrar una mayor equivalencia semántica. Más allá de esta limitación, se corroboró la estructura pentafactorial (hipótesis 1), se verificó que las cargas secundarias no fueron de magnitud significativa (hipótesis 2), y se corroboró la superioridad del modelo de cinco factores por sobre los modelos rivales (hipótesis 3 ).
Otro aspecto importante a destacar es que la influencia de un factor general sobre todos los ítems no recibe respaldo empírico, pues la regulación conductual de las emociones debería conceptualizarse sobre la base de dimensiones relacionadas, pero diferentes. Tal como señalan Kraaij y Garnefski (2019), a pesar de las correlaciones existentes entre las diferentes estrategias conductuales, se observa un elevado nivel de varianza única en cada factor.

Respecto a las evidencias de validez por su relación con otras variables, el análisis de regresión lineal indica que las estrategias conductuales examinadas por el BERQ predicen significativamente los niveles de distrés y eutrés. Al igual que en el estudio original (Kraaij \& Garnefski, 2019), escalas teóricamente más adaptativas - como distraerse y enfrentar activamente - mostraron una relación directa y significativa con el eutrés, mientras que escalas teóricamente menos adaptativas - como retirarse e ignorar el problema - mostraron una relación directa y significativa con el distrés. Estos resultados brindan evidencias favorables para las hipótesis 4 y 5 , aunque es importante profundizar en futuros estudios sobre algunos aspectos.

En primer lugar, es llamativo que la búsqueda de apoyo no presente relaciones significativas con el eutrés o el distrés. Diferentes investigaciones indican que el apoyo social es una fuente de bienestar y un factor protector del estrés (Medrano et al., 2018). De hecho, estudios metaanalíticos recientes indican que la comunicación social positiva con familiares y amigos reduce la probabilidad de enfermarse o tener un padecimiento mental (Harandi et al., 2017). Llamativamente, en el estudio original la escala de búsqueda de apoyo social tampoco mostró correlaciones significativas con depresión o ansiedad cuando se controlaba el efecto de las otras escalas mediante correlaciones parciales (Kraaij \& Garnefski, 2019). Esto indica que el comportamiento de búsqueda de apoyo debería analizarse con mayores matices, pues la búsqueda excesiva de contención y ayuda frente a situaciones de estrés puede generar un patrón de dependencia y transformarse en una fuente de malestar emocional; y a la vez la búsqueda excesiva de apoyo social puede generar temor al abandono y a la separación interpersonal, deteriorar la autonomía, promover conductas de sumisión social, y aumentar los sentimientos de vulnerabilidad e impotencia (Maccaferri et al., 2020). Debería analizarse en futuras investigaciones si existe una relación no lineal entre la búsqueda de apoyo y la salud mental. 
Otro aspecto llamativo es que solo las estrategias de distracción y retirada presentaron correlaciones "espejo". Por ejemplo, puntajes elevados en la escala de retirada se asocian a mayores niveles de distrés y menores de eutrés. Esta asociación simultánea con el distrés y eutrés no se aprecia en las escalas restantes. El enfrentamiento activo solo se asocia al eutrés y no al distrés, mientras que ignorar el problema solo se asocia al distrés, y no al eutrés.

Por otro lado, el coeficiente $\omega$ obtenido en cada caso presentó magnitudes que permiten concluir que la varianza verdadera asociada al constructo es significativa, lo cual da soporte a la hipótesis 6 .

En general, el presente estudio posee implicaciones prácticas relevantes para el ámbito universitario. Tal como demuestran investigaciones previas, el papel de las emociones es crítico para promover procesos de aprendizajes efectivos y satisfactorios. Al respecto, se ha observado que estudiantes con dificultades en el proceso de regulación emocional presentan bajas creencias de autoeficacia (Medrano, Flores-Kanter et al., 2016), menor satisfacción académica (Trógolo \& Medrano, 2012), menor engagement académico (Kwon et al., 2018) y, consecuentemente, menor rendimiento (Seibert et al., 2017). En este punto, cabe destacar que la regulación emocional es un constructo clave para promover un comportamiento académico óptimo, pero también para resguardar la salud mental de los estudiantes, y más si se tiene en cuenta que durante el tránsito de los estudios universitarios se debe atravesar por situaciones de presión académica y cambios vitales que pueden derivar en problemas emocionales si no se cuenta con un adecuado repertorio de regulación emocional (Compas et al., 2013; Medrano \& Trógolo, 2016).

Hasta la fecha, en el contexto latinoamericano los programas de entrenamiento en regulación de emociones se han centrado principalmente en variables cognitivas; no obstante, a partir del presente trabajo se abre un abanico de nuevas posibilidades prácticas. Por primera vez se cuenta con un instrumento diseñado para examinar variables conductuales de regulación emocional adaptado específicamente al contexto latinoamericano, lo cual permitirá: (a) la evaluación con fines de detección y diagnóstico de dificultades en la regulación conductual de las emociones en estudiantes universitarios, (b) medir la eficacia de programas de intervención que busquen a promover la regulación emocional en universitarios, y (c) desarrollar nuevas investigaciones que tengan como objetivo examinar el papel de la regulación conductual de las emociones. En conjunto, dichas acciones permitirán desarrollar prácticas con evidencia científica que puedan mejorar los procesos de aprendizaje y promover el bienestar psicológico de los estudiantes universitarios.

Un aspecto especialmente importante a considerar a partir de los resultados obtenidos es que debe contemplarse que ciertas estrategias de regulación emocional se asocian a una regulación del malestar emocional (Aldao et al., 2010; Coiro et al., 2017; Kaminski et al., 2006; Pritchard et al., 2007), mientras que otras se asocian en mayor medida a la promoción del bienestar (Horwitz et al., 2011; Joorman \& Stanton, 2016; Mahmoud et al., 2015). A partir de los resultados del presente estudio, se refuerza la importancia de desarrollar programas de intervención que integren estrategias para promover emociones positivas - p. ej., distraerse y enfrentar activamente - y regular emociones negativas — p. ej., ignorar el problema y retirada-

Asimismo, los resultados obtenidos llevan a repensar las intervenciones centradas en promover redes de apoyo social en los estudiantes universitarios (Medrano \& Orlando, 2008), pues la búsqueda de apoyo social puede asociarse, o no, a una regulación adecuada de las emociones. Concretamente, los hallazgos del presente estudio parecerían indicar que la búsqueda de apoyo puede resultar una estrategia conductual adecuada para disminuir el estrés solo en la medida en que promueva un afrontamiento activo del problema, de lo contrario, puede transformarse en una fuente de dependencia (Banerjee, 2008; Strenna et al., 2009).

Con todo, el presente estudio muestra algunas limitaciones. En primer lugar, al tratarse de una muestra no representativa, no es posible extender los hallazgos a todos los estudiantes peruanos; $y$ en segundo lugar, no fue posible ejecutar un análisis de invarianza de medición respecto al sexo, debido a la cantidad de personas por grupo, sin embargo, se comparó los coeficientes de confiabilidad entre hombres y mujeres como una aproximación descriptiva a la diferencia en cuanto a la estimación del error de medición.

Finalmente, pese a estas limitaciones, el trabajo realizado constituye un punto de partida para el desarrollo de investigaciones que tengan como objetivo examinar el rol de las conductas involucradas en la RE en países de habla hispana, siendo esta la primera vez que se analiza una versión en español del BERQ. Además, son resultados son 
de gran importancia para la población de universitarios de Perú, ya que a partir de este trabajo se podría continuar con estudios psicométricos que permitan consolidar al BERQ en español como un instrumento válido y confiable que permita indagar el rol de estrategias conductuales de RE en el comportamiento académico, así como extender su estudio a estudiantes universitarios de otros países hispanohablantes.

\section{Referencias}

Aldao, A., Nolen-Hoeksema, S., \& Schweizer, S. (2010). Emotion-regulation strategies across psychopathology: A meta-analytic review. Clinical Psychology Review, 30(2), 217-237. https://doi.org/10.1016/j.cpr.2009.11.004

American Educational Research Association, American Psychological Association, \& National Council on Measurement in Education [AERA/APA/NCME] (2014). Standards for educational and psychological testing. American Psychological Association.

Asparouhov, T., \& Muthén, B. (2009). Exploratory structural equation modeling. Structural Equation Modeling: A Multidisciplinary Journal, 16(3), 397-438. https://doi. org/10.1080/10705510903008204

Ato, M., López, J., \& Benavente, A. (2013). Un sistema de clasificación de los diseños de investigación en psicología. Anales de Psicología, 29(3), 1038-1059. https://doi. org/10.6018/analesps.29.3.178511

Banerjee, R. A. (2008). Social cognition and anxiety in children. En C. Sharp, P. Fonagy y I. Goodyer (eds.), Social cognition and developmental psychopathology (pp. 239269). Oxford University Press.

Barlow, D. H., Allen, L. B., \& Choate, M. L. (2004). Toward a unified treatment for emotional disorders. Behavior Therapy, 35(2), 205-230. https://doi.org/10.1016/S00057894(04)80036-4

Coiro, M. J., Bettis, A. H., \& Compas, B. E. (2017). College students coping with interpersonal stress: Examining a control-based model of coping. Journal of American
College Health, 65(3), 177-186. https://doi.org/10.1080 /07448481.2016.1266641

Compas, B. E., Jaser, S. S., Dunbar, J. P., Watson, K. H., Bettis, A. H., Gruhn, M. A., \& Williams, E. K. (2013). Coping and emotion regulation from childhood to early adulthood: Points of convergence and divergence. Australian Journal of Psychology, 66(2), 71-81. https://doi.org/10.1111/ajpy.12043

Cuijpers, P., Cristea, I. A., Ebert, D. D., Koot, H. M., Auerbach, R. P., Bruffaerts, R., \& Kessler, R. C. (2016). Psychological treatment of depression in college students: a metaanalysis. Depression and Anxiety, 33(5), 400-414. https://doi.org/10.1002/da.22461

DiStefano, C., Liu, J., Jiang, N., \& Shi, D. (2018). Examination of the weighted root mean square residual: Evidence for trustworthiness? Structural Equation Modeling: A Multidisciplinary Journal, 25(3), 453-466. https://doi.org/10.1080/10705511.2017.1390394

Dominguez-Lara, S. (2018). Propuesta de puntos de corte para cargas factoriales: una perspectiva de fiabilidad de constructo. Enfermería Clínica, 28(6), 401-402. https://doi.org/10.1016/j.enfcli.2018.06.002

Dominguez-Lara, S., \& Merino-Soto, C. (2018). Evaluación de las malas especificaciones en modelos de ecuaciones estructurales. Revista Argentina de Ciencias del Comportamiento, 10(2), 19-24. https://doi. org/10.32348/1852.4206.v10.n2.19595

Dominguez-Lara, S., Merino-Soto, C., \& Navarro-Loli, J. (2018). Comparación de coeficientes alfa basada en intervalos de confianza. Educación Médica, 19(1), 70. https://doi.org/10.1016/j.edumed.2017.03.025

Ellis, P. D. (2010). The essential guide to effect sizes: An introduction to statistical power, meta-analysis and the interpretation of research results. Cambridge University Press.

Ferguson, C. J. (2009). An effect size primer: a guide for clinicians and researchers. Professional Psychology: 
Research and Practice, 40(5), 532-538. https://doi. org/10.1037/a0015808

Fleming, J., \& Merino, C. (2005). Medidas de simplicidad y ajuste factorial: Un enfoque para la construcción y revisión de escalas derivadas factorialmente. Revista de Psicología, 23(2), 252-266. https://doi.org/10.18800/ psico.200502.002

Fornell, C., \& Larcker, D. F. (1981). Evaluating structural equation models with unobservable variables and measurement error. Journal of Marketing Research, 18, 39-50. https://doi.org/10.1177/002224378101800104

Garnefski, N., Kraaij, V., \& Spinhoven, P. (2001). Negative life events, cognitive emotion regulation and emotional problems. Personality and Individual Differences, 30(2), 1311-1327. https://doi.org/10.1016/ S0191-8869(00)00113-6

Gravetter, F., \& Wallnau, L. (2014). Essentials of Statistics for the Behavioral Sciences. Wadsworth.

Gross, J. J. (2008). Emotion regulation. En M. Lewis, J. M. Haviland-Jones y L. F. Barrett (eds.), Handbook of emotions (pp. 497-512). The Guilford Press.

Gross, J. J. (2015). Emotion regulation: current status and future prospects. Psychological Inquiry, 26, 1- 26. https://doi.org/10.1080/1047840X.2014.940781

Hair, J. F., Black, B., Babin, B., Anderson, R. E., \& Tatham, R. L. (2010). Multivariate data analysis (7. ${ }^{\mathrm{a}}$ ed.). Prentice Hall.

Hambleton, R. K. (2005). Issues, designs, and technical guidelines for adapting tests into multiple languages and cultures. En R. K. Hambleton, P. F. Merenda y C. D. Spielberger (eds.), Adapting educational and psychological tests for cross-cultural assessment (pp. 3-38). Lawrence Erlbaum Associates.

Harandi, T. F., Taghinasab, M. M., \& Nayeri, T. D. (2017). The correlation of social support with mental health: A meta-analysis. Electronic Physician, 9(9), 5212-5222. https://doi.org/10.19082/5212

Horwitz, A. G., Hill, R. M., \& King, C. A. (2011). Specific coping behaviors in relation to adolescents depression and suicidal ideation. Journal of Adolescence, 34(5), 10771085. https://doi.org/10.1016/j.adolescence.2010.10.004

Hu, L., \& Bentler, P. M. (1998). Fit indices in covariance structure modeling: Sensitivity to underparameterized model specification. Psychological Methods, 3(4), 424453. https://doi.org/10.1037/1082-989X.3.4.424

Hunsley, J., \& Marsh, E.J. (2008). Developing criteria for evidence-based assessment: An introduction to assessment that work. En J. Hunsley y E. J. Marsh (eds.), $A$ guide to assessments that work (pp. 3-14). Oxford University Press.

Joormann, J., \& Stanton, C. H. (2016). Examining emotion regulation in depression: a review and future directions. Behaviour Research and Therapy, 86, 35-49. https://doi. org/10.1016/j.brat.2016.07.007

Joormann, J., Yoon, K. L., \& Siemer, M. (2010). Cognition and emotion regulation. En A. M. Kring y D. M. Sloan (eds.), Emotion regulation and psychopathology: A transdiagnostic approach to etiology and treatment (pp. 174-203). The Guilford Press.

Kaminski, L., Robertson, M., \& Dewey, D. (2006). Psychological correlates of depression in children with recurrent abdominal pain. Journal of Pediatric Psychology, 31(9), 956-966. https://doi.org/10.1093/ jpepsy/jsj103

Kraaij, V., \& Garnerfski, N. (2019). The Behavioral Emotion Regulation Questionnaire: Development psychometric properties and relationships with emotional problems and the Cognitive Emotion Regulation Questionnaire. Personality and Individual Differences, 137, 56-61. https://doi.org/10.1016/j.paid.2018.07.036

Kwon, K., Kupzyk, K., \& Benton, A. (2018). Negative emotionality, emotion regulation, and achievement: 
Cross-lagged relations and mediation of academic engagement. Learning and Individual Differences, 67, 33-40. https://doi.org/10.1016/j.lindif.2018.07.004

Liu, D. Y., \& Thompson, R. J. (2017). Selection and implementation of emotion regulation strategies in major depressive disorder: An integrative review. Clinical Psychology Review, 57, 183-194. https://doi. org/10.1016/j.cpr.2017.07.004

Lloret-Segura, S., Ferreres-Traver, A., Hernández-Baeza, A., \& Tomás-Marco, I. (2014). El análisis factorial exploratorio de los items: una guía práctica, revisada y actualizada. Anales de Psicología, 30(3), 1151-1169. https://doi. org/10.6018/analesps.30.3.199361

Maccaferri, G. E., Dunker-Scheuner, D., De Roten, Y., Despland, J. N., Sachse, R., \& Kramer, U. (2020). Psychotherapy of dependent personality disorder: The relationship of patient-therapist interactions to outcome. Psychiatry, 83(2), 179-194. https://doi.org/10.1080/0033 2747.2019.1675376

Mahmoud, J. S. R., Staten, R. “Topsy,” Lennie, T. A., \& Hall, L. A. (2015). The relationships of coping, negative thinking, life satisfaction, social support, and selected demographics with anxiety of young adult college students. Journal of Child and Adolescent Psychiatric Nursing, 28(2), 97-108. https://doi.org/10.1111/jcap.12109

Mardia, K. (1970). Measures of multivariate skewness and kurtosis with applications. Biometrika, 57(3), 519-530. https://doi.org/10.2307/2334770

Mauss, I. B., Levenson, R. W., McCarter, L., Wilhelm, F. H., \& Gross, J. J. (2005). The tie that binds? Coherence among emotion experience, behavior, and physiology. Emotion, 5(2), 175-190. https://doi. org/10.1037/1528-3542.5.2.175

McDonald, R. P., \& Ho, M.-H. R. (2002). Principles and practice in reporting structural equation analyses. Psychological Methods, 7(1), 64-82. https://doi. org/10.1037/1082-989X.7.1.64
Medrano, L. A., \& Orlando, F. (2008). Autoeficacia social en ingresantes universitarios: Su relación con el rendimiento y la deserción académica. Revista de Psicología General y Aplicada, 61(4), 369-384. https://dialnet. unirioja.es/servlet/articulo?codigo $=2982857$

Medrano, L. A., \& Trógolo, M. (2016). Construct validity of the Difficulties in Emotion Regulation Scale: Further evidence using confirmatory factor analytic approach. Abnormal and Behavioural Psychology, 2(117). https://doi.org/10.4172/2472-0496.1000117

Medrano, L. A., Flores-Kanter, E., Moretti, L., \& Pereno, G. L. (2016). Effects of induction of positive and negative emotional states on academic self-efficacy beliefs in college students. Psicología Educativa, 22(2), 135-141. https://doi.org/10.1016/j.pse.2015.03.003

Medrano, L. A., Muñoz-Navarro, R., \& Cano-Vindel, A. (2016). Procesos cognitivos y regulación emocional: Aportes desde una aproximación psicoevolucionista. Ansiedad y Estrés, 22, 47-54. https://doi.org/10.1016/j. anyes.2016.11.001

Medrano, L. A., Ortiz, A., Flores-Kanter, P. E., DominguezLara, S. A., \& Gibelli, S. (2018). Medición de recursos personales socio-cognitivos en organizaciones: Análisis psicométricos en trabajadores argentinos. Revista Evaluar, 18(2), 1-16. https://doi.org/10.35670/16674545.v18.n2.20799

Moscoso, M. S., Lengacher, C. A., \& Knapp, M. (2012). Estructura factorial del Inventario Multicultural de la Depresión, Estado-Rasgo: rol de las emociones positivas en la depresión. Persona, 15, 115-136. https://doi. org/10.26439/persona2012.n015.129

Muñiz, J., Elosua, P., \& Hambleton, R. K. (2013). Directrices para la traducción y adaptación de los test: segunda edición. Psicothema, 25(2), 151-157. https:// doi.org/10.7334/psicothema2013.24

Muthén, L. K., \& Muthén, B. O. (1998-2015). Mplus User's guide (7. ${ }^{\mathrm{a}}$ ed.). Muthén \& Muthén. 
Pérez, E. \& Medrano, L. (2010). Análisis Factorial Exploratorio: Bases Conceptuales y Metodológicas. Revista Argentina de Ciencias del Comportamiento, 2(1), 58-66. https://doi.org/10.32348/1852.4206.v2.n1.15924

Ponterotto, J., \& Charter, R. (2009). Statistical extensions of Ponterotto and Ruckdeschel's (2007) reliability matrix for estimating the adequacy of internal consistency coefficients. Perceptual and Motor Skills, 108(3), 878886. https://doi.org/10.2466/PMS.108.3.878-886

Pritchard, M. E., Wilson, G. S., \& Yamnitz, B. (2007). What predicts adjustment among college students? A longitudinal panel study. Journal of American College Health, 56(1), 15-21. https://doi.org/10.3200/JACH.56.1.15-22

Rodríguez, A., Reise, S. P., \& Haviland, M. G. (2016). Applying bifactor statistical indices in the evaluation of psychological measures. Journal of Personality Assessment, 98(3), 223-237. https://doi.org/10.1080/002 23891.2015.1089249

Rodríguez, M., \& Ruiz, M. (2008). Atenuación de la asimetría y de la curtosis de las puntuaciones observadas mediante transformaciones de variables: Incidencia sobre la estructura factorial. Psicológica, 29, 205-227. https://www.uv.es/psicologica/articulos2.08/6RODRI GUEZ.pdf

Saris, W. E., Satorra, A., \& van der Veld, W. M. (2009). Testing structural equation models or detection of misspecifications? Structural Equation Modeling: A Multidisciplinary Journal, 16(4), 561-582. https://doi. org/10.1080/10705510903203433

Scherer, M. R. (1984). On the nature and function of emotion: A component process approach. En K. R. Scherer y P. E. Ekman (eds.), Approaches to emotion (pp. 293317). Lawrence Erlbaum Associates.

Seibert, G. S., Bauer, K. N., May, R. W., \& Fincham, F. D. (2017). Emotion regulation and academic underperformance: The role of school burnout. Learning and Individual Differences, 60, 1-9. https://doi. org/10.1016/j.lindif.2017.10.001
Sijtsma, K. (2009). On the use, the misuse, and the very limited usefulness of Cronbach's alpha. Psychometrika, 74(1), 107-120. https://doi.org/10.1007/s11336-008-9101-0

Smits, I.A. M., Timmerman, M.E., Barelds, D.P.H., \& Meijer, R. R. (2015). The Dutch symptom checklist-90-revised: is the use of the subscales justified? European Journal of Psychological Assessment, 31(4), 263-271. https://doi. org/10.1027/1015-5759/a000233

Strenna, L., Chahraoui, K., \& Vinay, A. (2009). Santé psychique chez les étudiants de première année d'école supérieure de commerce: liens avec le stress de l'orientation professionnelle, l'estime de soi et le coping. L'Orientation Scolaire et Professionnelle, 38(2), 183204. https://doi.org/10.4000/osp.1902

Tamir, M. (2016). Why do people regulate their emotions? A taxonomy of motives in emotion regulation. Personality and Social Psychology Review, 20(3), 199-222. https:// doi.org/10.1177/108886831558632

Trógolo, M., \& Medrano, L. A. (2012). Personality traits, difficulties in emotion regulation and academic satisfaction in a sample of argentine college students. International Journal of Psychological Research, 5(2), 30-39. https://doi.org/10.21500/20112084.734

Völlink, T., Bolman, C., Dehue, F., \& Jacobs, N. (2013). Coping with cyberbullying: differences between victims, bully-victims and children not involved in bullying. Journal of Community and Applied Social Psychology, 23(1), 7-24. https://doi.org/10.1002/casp.2142

Völlink, T., Bolman, C., Eppingbroek, A., \& Dehue, F. (2013). Emotion-focused coping worsens depressive feelings and health complaints in cyberbullied children. Journal of Criminology, 2013, 416976. https://doi. org $/ 10.1155 / 2013 / 416976$

Wahed, W. Y. A., \& Hassan, S. K. (2017). Prevalence and associated factors of stress, anxiety and depression among medical Fayoum University students. Alexandria Journal of Medicine, 53(1), 77-84. https://doi.org/10.1016/j. ajme.2016.01.005 
West, S. G., Taylor, A. B., \& Wu, W. (2012). Model fit and model selection in structural equation modeling. En R. H. Hoyle (ed.), Handbook of Structural Equation Modeling (pp. 209-231). The Guilford Press.

Zinbarg,R.E., Yovel,I., Revelle,W.,\&McDonald,R.P.(2006). Estimating generalizability to a latent variable common to all of a scale's indicators: A comparison of estimators for $\omega \mathrm{h}$. Applied Psychological Measurement, 30(2), 121144. https://doi.org/10.1177/0146621605278814

Zwick, W., \& Velicer, W., (1986). Comparison of five rules for determining the number of components to retain. Psychological Bulletin, 99(3), 432-442. https:// doi.org/10.1037/0033-2909.99.3.432 